\title{
On the Use of Transeunt Triangles to Synthesize Fixed-Polarity Reed-Muller Expansions of Functions
}

\author{
Jon T. Butler \\ Dept. of Electr. and Comp. Eng. \\ Naval Postgraduate School \\ Monterey, CA 93943-5121 U.S.A. \\ Jon_Butler@msn.com \\ Svetlana Yanushkevich \\ University of Calgary \\ ECE Department \\ Calgary, AB T2N 1N4 CANADA \\ syanshk@ucalgary.ca
}

\author{
Gerhard W. Dueck \\ University of New Brunswick \\ Faculty of Computer Science \\ Frederiction, NB E3B 5A3 CANADA \\ gdueck@unb.ca \\ Vlad P. Shmerko \\ University of Calgary \\ ECE Department \\ Calgary, AB T2N 1N4 CANADA \\ vshmerko@ucalgary.ca
}

\begin{abstract}
The transeunt triangle was originally proposed by Suprun [19] as the basis of an algorithm for synthesizing fixed-polarity Reed-Muller (FPRM) expansions of symmetric functions. However, he provided no proof that this technique produced the correct FPRM expansion. We provide such a proof, thus establishing the validity of the transeunt triangle technique. Further, we show the extent to which the transeunt triangle reduces the computational work needed. Because of the efficiency of the transeunt triangle, we are able to do experimental studies on sets of n-variable symmetric functions for large values of n never before achievable. For example, we show that a surprisingly large percentage of symmetric functions (35\% for large n) are optimally realized by just two $($ of $n+1)$ polarities. This is verified by exhaustive enumeration of symmetric functions with up to 31 variables and by large sample sets $(1,000,000)$ of symmetric functions with up to 100 variables. This suggests that even greater efficiency can be achieved through a heuristic that restricts the polarities to one or both of the favored polarities.
\end{abstract}

\section{Introduction}

Much is known about sum-of-product expressions in which sum corresponds to Exclusive-OR and product corresponds to the AND of variables or complements of variables [4]. For example, such EXOR sum-of-product expressions require, on the average, fewer product terms than OR sum-of-products [14]. Also, such structures are known to be easily testable [13]. AND-EXOR circuits have been used in arithmetic, error correcting, and telecommunications applications [15], [16].

This paper focuses on the fixed polarity Reed-Muller (FPRM) expansion [21, 22]. In an FPRM expansion of a given function $f(X)$, every variable appears either complemented or uncomplemented; never in both forms. If all variables are uncomplemented (complemented), the FPRM expansion is called the Positive (Negative) Polarity ReedMuller or PPRM (NPRM) form. FPRM expansions are unique [5]. Thus, only one representation exists for the PPRM or NPRM or indeed any FPRM of $f(X)$. This leads to the question of which of the $n+1$ FPRM's produce the fewest product terms $[10,11,17]$.

This has inspired study of the FPRM expansion of an important class of functions, symmetric functions. In 1995, Suprun [20] showed the use of the transeunt triangle in converting between various FPRMs. The origin of the transeunt triangle is unknown. In 1986, Green [7] p. 141, mentioned that a "triangular structure" could produce the transformation produced by a transform matrix. The transeunt triangle has shown to produce a fast $\left(\mathrm{O}\left(n^{3}\right)\right.$ time complexity) and compact $\left(\mathrm{O}\left(n^{2}\right)\right.$ storage complexity) algorithm for synthesis of symmetric functions [2]. Further, transeunt triangles have simple structures and can be generated from only a few basic triangles [3].

There have been many studies of symmetric functions dating back to the early history of switching theory. Interest in this important area has continued to the present. For example, many benchmark functions are symmetric. It 


\section{Report Documentation Page}

Public reporting burden for the collection of information is estimated to average 1 hour per response, including the time for reviewing instructions, searching existing data sources, gathering and maintaining the data needed, and completing and reviewing the collection of information. Send comments regarding this burden estimate or any other aspect of this collection of information, including suggestions for reducing this burden, to Washington Headquarters Services, Directorate for Information Operations and Reports, 1215 Jefferson Davis Highway, Suite 1204, Arlington VA 22202-4302. Respondents should be aware that notwithstanding any other provision of law, no person shall be subject to a penalty for failing to comply with a collection of information if it does not display a currently valid OMB control number.

\begin{tabular}{|l|l|l|}
\hline 1. REPORT DATE & \multirow{2}{*}{$\begin{array}{l}\text { 2. REPORT TYPE } \\
\text { MAY } 2009\end{array}$} & 5. DATES COVERED \\
\hline $\begin{array}{l}\text { 4. TITLE AND SUBTITLE } \\
\text { On the Use of Transeunt Triangles to Synthesize Fixed-Polarity } \\
\text { Reed-Muller Expansions of Functions }\end{array}$ & 5b. GRANT NUMBER \\
\cline { 2 - 3 } & 5c. PROGRAM ELEMENT NUMBER \\
\hline 6. AUTHOR(S) & 5d. PROJECT NUMBER \\
\cline { 2 - 3 } & 5e. TASK NUMBER \\
\cline { 2 - 3 } & 5f. WORK UNIT NUMBER \\
\hline $\begin{array}{l}\text { 7. PERFORMING ORGANIZATION NAME(S) AND ADDRESS(ES) } \\
\text { Naval Postgraduate School,Department of Electrical and Computer } \\
\text { Engineering,Monterey,CA,93943 }\end{array}$ & $\begin{array}{l}\text { 8. PERFORMING ORGANIZATION } \\
\text { REPORT NUMBER }\end{array}$ \\
\hline 9. SPONSORING/MONITORING AGENCY NAME(S) AND ADDRESS(ES) & \\
\hline
\end{tabular}

12. DISTRIBUTION/AVAILABILITY STATEMENT

Approved for public release; distribution unlimited.

13. SUPPLEMENTARY NOTES

14. ABSTRACT

The transeunt triangle was originally proposed by Suprun [19] as the basis of an algorithm for synthesizing fixed-polarity Reed-Muller (FPRM) expansions of symmetric functions. However, he provided no proof that this technique produced the correct FPRM expansion. We provide such a proof, thus establishing the validity of the transeunt triangle technique. Further, we show the extent to which the transeunt triangle reduces the computational work needed. Because of the efficiency of the transeunt triangle, we are able to do experimental studies on sets of $n$-variable symmetric functions for large values of $n$ never before achievable. For example, we show that a surprisingly large percentage of symmetric functions (35\% for large $n)$ are optimally realized by just two $($ of $n+1)$ polarities. This is verified by exhaustive enumeration of symmetric functions with up to 31 variables and by large sample sets $(1,000,000)$ of symmetric functions with up to 100 variables. This suggests that even greater efficiency can be achieved through a heuristic that restricts the polarities to one or both of the favored polarities.

15. SUBJECT TERMS

16. SECURITY CLASSIFICATION OF:

\begin{tabular}{c|c|c}
$\begin{array}{c}\text { a. REPORT } \\
\text { unclassified }\end{array}$ & $\begin{array}{c}\text { b. ABSTRACT } \\
\text { unclassified }\end{array}$ & $\begin{array}{c}\text { c. THIS PAGE } \\
\text { unclassified }\end{array}$
\end{tabular}

17. LIMITATION OF ABSTRACT
18. NUMBER OF PAGES

8 19a. NAME OF RESPONSIBLE PERSON unclassified 
is known [6] that symmetric functions are very difficult to minimize by Quine-McCluskey-like algorithms. Therefore, they are ideal benchmark functions. That is, their minimal forms are known, but algorithms have difficulty finding them [12]. Although symmetric functions represent a minority of the set of switching functions, they often appear in logic design. For example, basic gates, AND, OR, EXOR are symmetric, as are parity checkers and majority gates. It is known that any logic function has a symmetric function realization in which certain variables are repeated [1].

While this paper focuses on binary-valued functions, it is worth noting that interesting results have been produced for multiple-valued functions. For example, Green [9] has derived Reed-Muller expansions for four-valued functions. A three-valued map has been demonstrated by Green [8] to be useful in the synthesis of mixed-polarity binary Reed-Muller expansions (both complemented and uncomplemented variables occur).

This paper is organized as follows. The next section introduces the synthesis problem and the transeunt triangle. The third section proves that the transeunt triangle can be used as a basis for synthesizing FPRM expansions. The fourth section discusses the algorithm's time and space complexity. The fifth section shows experimental results, and the final section contains concluding remarks.

\section{Notation and Fundamental Results}

\subsection{Symmetric Functions}

Definition 2.1 Let $\quad S_{A}^{n} \quad$ be $\quad a \quad$ (totally) symmetric function that is 1 iff $m$ of its $n$ variables are 1 , where $m \in A \subseteq\{0,1, \ldots, n\}$.

Example 2.1 $S_{\{0,1,2\}}^{3}$ is a symmetric function that is 1 iff 0,1 , and 2 of its variables are 1. It is the NAND of three variables.

(End of Example)

Definition 2.2 The carrier vector $\Lambda\left(S_{A}^{n}\right)=$ $\left[\alpha_{0}, \alpha_{1}, \ldots, \alpha_{n}\right]$ of symmetric function $S_{A}^{n}$ is a binary $n+1$-tuple, where $\alpha_{m}$ is 1 if $m \in A$ and is 0 otherwise. $\alpha_{m}$ is called an $\mathbf{a}-$ number [18].

Example 2.2 The carrier vector of $S_{\{0,1,2\}}^{3}$ is $[1,1,1,0]$. (End of Example)

\subsection{Reed-Muller Expansions}

The Reed-Muller expansion of a general switching function $f$ is the exclusive OR of product terms of variables or their complements. The term fixed polarity is used to describe Reed-Muller expansions in which each variable appears uncomplemented or complemented, and never in both forms. For symmetric functions, the FPRM expansion has a restricted form.
Definition 2.3 The polarity $p$ Reed-Muller expansion $R M_{p}\left(S_{A}^{n}\right)$ of symmetric function $S_{A}^{n}$ is

$$
\begin{gathered}
R M_{p}\left(S_{A}^{n}\right)=c_{0,0}^{p} \oplus c_{0,1}^{p} \sum_{i=1}^{p} \bar{x}_{i} \oplus c_{1,0}^{p} \sum_{j=p+1}^{n} x_{j} \oplus \ldots \\
\oplus c_{u, c}^{p} \bar{x}_{i_{1}} \bar{x}_{i_{2}} \ldots \bar{x}_{i_{c}} x_{j_{1}} x_{j_{2}} \ldots x_{j_{u}} \oplus \ldots \\
1 \leq i_{1}<i_{2}<\ldots<i_{c} \leq p \\
p+1 \leq j_{1}<j_{2}<\ldots<j_{u} \leq n \\
\oplus c_{n-p, p}^{p} \bar{x}_{1} \bar{x}_{2} \ldots \bar{x}_{p} x_{p+1} x_{p+2} \ldots x_{n}
\end{gathered}
$$

where $\sum \oplus$ is the exclusive OR of products of uncomplemented variables and complemented variables, and $c_{u, c}^{p} \in$ $\{0,1\}$ is its coefficient, such that there are exactly u uncomplemented variables and exactly c complemented variables.

As will be seen later, $c_{u, c}^{p}$ cannot, in general, be chosen arbitrarily. Because a symmetric function is unchanged by a permutation of variables, it makes no difference which variables in $R M_{p}$ appear complemented. To simplify the notation, however, we assume that $x_{1}, x_{2}, \ldots$, and $x_{p}$ appear complemented in $R M_{p}$, while $x_{p+1}, x_{p+2}, \ldots$, and $x_{n}$ appear uncomplemented.

Example 2.3 For $S_{\{0,1,2\}}^{3}$,

$$
\begin{aligned}
& R M_{0}\left(S_{\{0,1,2\}}^{3}\right)=1 \oplus x_{1} x_{2} x_{3}, \\
& R M_{1}\left(S_{\{0,1,2\}}^{3}\right)=1 \oplus x_{2} x_{3} \oplus \bar{x}_{1} x_{2} x_{3}, \\
& R M_{2}\left(S_{\{0,1,2\}}^{3}\right)=1 \oplus x_{3} \oplus\left(\bar{x}_{1} x_{3} \oplus \bar{x}_{2} x_{3}\right) \oplus \bar{x}_{1} \bar{x}_{2} x_{3}, \text { and } \\
& R M_{3}\left(S_{\{0,1,2\}}^{3}\right)=\left(\bar{x}_{1} \oplus \bar{x}_{2} \oplus \bar{x}_{3}\right) \oplus\left(\bar{x}_{1} \bar{x}_{2} \oplus \bar{x}_{1} \bar{x}_{3} \oplus \bar{x}_{2} \bar{x}_{3}\right) \oplus \bar{x}_{1} \bar{x}_{2} \bar{x}_{3} .
\end{aligned}
$$

(End of Example)

Definition 2.4 The coefficient matrix $C_{p}\left(S_{A}^{n}\right)$ of the polarity $p$ Reed-Muller expansion of symmetric function $S_{A}^{n}$ is a $(p+1) \times(n-p+1)$ matrix $\left[c_{u, c}^{p}\right]$, where $c_{u, c}^{p}$ is an $R M_{p}\left(S_{A}^{n}\right)$ coefficient.

Example 2.4 For our running example, 


$$
\begin{aligned}
& C_{0}\left(S_{\{0,1,2\}}^{3}\right)=c \uparrow 0\left[\begin{array}{llll}
1 & 0 & 0 & 1
\end{array}\right] \\
& \underset{u}{0} \mathbb{1}^{2} \quad 3 \\
& \begin{aligned}
C_{2}\left(S_{\{0,1,2\}}^{3}\right)=c^{4} & \begin{array}{c}
2 \\
1 \\
0
\end{array}\left[\begin{array}{ll}
0 & 1 \\
0 & 1 \\
1 & 1
\end{array}\right] \\
& \underset{u}{u}
\end{aligned}
\end{aligned}
$$$$
\begin{aligned}
C_{1}\left(S_{\{0,1,2\}}^{3}\right)=c^{\uparrow} & 1\left[\begin{array}{lll}
0 & 0 & 1 \\
0 & 0 & 1
\end{array}\right] \\
& \underset{u}{0}
\end{aligned}
$$

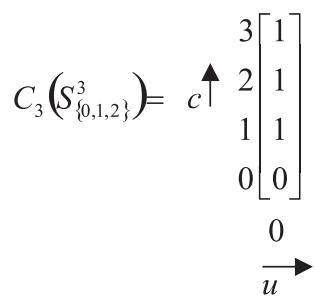

(End of Example)

\subsection{Transeunt Triangle}

Definition 2.5 The transeunt triangle $T\left(S_{A}^{n}\right)$ of symmetric function $S_{A}^{n}$ is a matrix $\left[t_{i, j}\right]$ of logic values, where

$$
\text { 1. } t_{i, j}=c_{n-j, 0}^{0} \quad i=0 \text { and } 0 \leq j \leq n,
$$

2. $t_{i, j}=t_{i-1, j} \oplus t_{i-1, j+1} \quad 1 \leq i \leq n$ and $0 \leq j \leq n-i$, and

$$
\text { 3. } t_{i, j} \text { is undefined otherwise, }
$$

where $c_{n-j, 0}^{0}$ is a coefficient in $C_{0}\left(S_{A}^{n}\right)$. Transeunt triangle $T\left(S_{A}^{n}\right)$ is said to be generated from $C_{0}\left(S_{A}^{n}\right)$.

Example 2.5 We construct $T\left(S_{\{0,1,2\}}^{3}\right)$ as follows. From Definition 2.5

$$
\begin{aligned}
& t_{0,0}, t_{0,1}, t_{0,2}, t_{0,3}=c_{3,0}^{0}, c_{2,0}^{0}, c_{1,0}^{0}, c_{0,0}^{0}=1,0,0,1 \\
& t_{1,0}, t_{1,1}, t_{1,2}=t_{0,0} \oplus t_{0,1}, t_{0,1} \oplus t_{0,2}, t_{0,2} \oplus t_{0,3}=1,0,1 \\
& t_{2,0}, t_{2,1}=t_{1,0} \oplus t_{1,1}, t_{1,1} \oplus t_{1,2}=1,1 \\
& t_{3,0}=t_{2,0} \oplus t_{2,1}=0 \\
& t_{i, j} \text { is undefined otherwise. Fig. } 1 \text { shows the complete }
\end{aligned}
$$

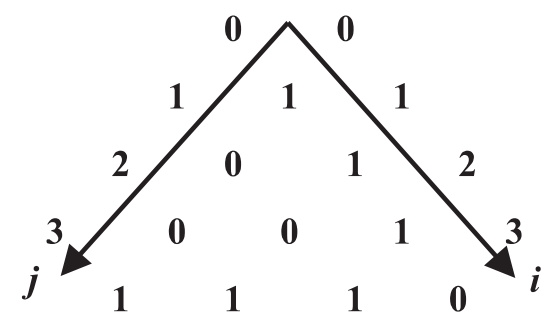

Figure 1. Transeunt triangle, $T\left(S_{\{0,1,2\}}^{3}\right)$.

triangle. Its orientation is chosen for the benefit of representing embedded submatrices, our next topic. Undefined elements are blank. Elements equal to the coefficients of $C_{0}\left(S_{\{0,1,2\}}^{3}\right)$ occur as the left side of the triangle.

(End of Example)
The specification of the transeunt triangle is a formalism that allows us to make statements about the coefficients of $R M_{p}\left(S_{A}^{n}\right)$. Of special interest are embedded submatrices of $T\left(S_{A}^{n}\right)$. For example, by construction, coefficient matrix $C_{0}\left(S_{\{0,1,2\}}^{3}\right)$ is an embedded submatrix of $T\left(S_{A}^{n}\right)$.

Definition 2.6 Given transeunt triangle $T\left(S_{A}^{n}\right)$ of symmetric function $S_{A}^{n}$, an embedded submatrix $M_{p}\left(S_{A}^{n}\right)$ is a $(p+1) \times(n-p+1)$ matrix of values $\left[m_{u, c}^{p}\right]$, such that

$$
m_{u, c}^{p}=t_{i, j}
$$

where

$$
\text { 1) } u=n-p-j \text { and 2) } c=p-i \text {. }
$$

For $p=0$, (3) and (4) yield $m_{n-j, 0}^{0}=t_{0, j}(c=-i$ implies $c=i=0$ ). From (2), we can write $m_{n-j, 0}^{0}=c_{n-j, 0}^{0}$, and

$$
M_{0}\left(S_{A}^{n}\right)=C_{0}\left(S_{A}^{n}\right) .
$$

That is, embedded submatrix $M_{0}\left(S_{A}^{n}\right)$ is identical to the coefficient matrix from which $T\left(S_{A}^{n}\right)$ was generated.

Example 2.6 Consider $S_{\{0,1,2\}}^{3}$. The four embedded submatrices associated with the transeunt triangle of $S_{\{0,1,2\}}^{3}$ are shown in Fig. 2.

(End of Example)
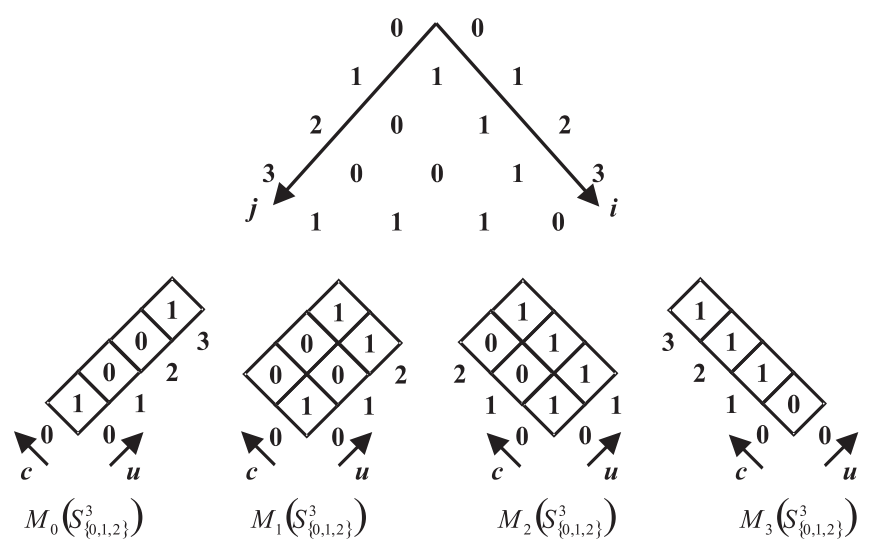

Figure 2. Submatrices $M_{p}\left(S_{\{0,1,2\}}^{3}\right)$ of transeunt triangle, $T\left(S_{\{0,1,2\}}^{3}\right)$.

Note that the origin $c=u=0$ in each embedded submatrix corresponds to a cell of the transeunt triangle in the diagonal (lowest row) of the transeunt triangle. Note also, that only diagonal elements occur in exactly one embedded submatrix. We show, in the next section, that these elements correspond to the carrier vector of $S_{A}^{n}$. All other elements occur in two or more embedded submatrices. Indeed, the origin $i=j=0$ of the transeunt triangle occurs in all embedded submatrices. 


\section{The Transeunt Triangle as the Basis for the Synthesis of FPRM Expansions}

Earlier, we observed that, when $p=0$, the coefficient matrix $C_{p}\left(S_{A}^{n}\right)$ corresponds to $M_{p}\left(S_{A}^{n}\right)$. This is equivalent to the statement that the left side of the transeunt triangle is the coefficient matrix of the PPRM expansion. Indeed, it is also true that the right side of the transeunt triangle is the coefficient matrix of the NPRM expansion. A formal proof of the latter two statements appeared in [19]. The following theorem extends this statement to all $p$. This was known by Suprun [20], but, as far as we know, no formal proof has been published.

Theorem 3.1 Let $C_{p}\left(S_{A}^{n}\right)$ be the coefficient matrix of the polarity $p$ Reed-Muller expansion of symmetric function $S_{A}^{n}$, and let $M_{p}\left(S_{A}^{n}\right)$ be the $p$-th embedded submatrix of the transeunt triangle for $S_{A}^{n}$. Then $C_{p}\left(S_{A}^{n}\right)=M_{p}\left(S_{A}^{n}\right)$.

\section{Proof}

We show that $C_{p-1}\left(S_{A}^{n}\right)=M_{p-1}\left(S_{A}^{n}\right)$ implies $C_{p}\left(S_{A}^{n}\right)=M_{p}\left(S_{A}^{n}\right)$, for $0<p \leq n$. The hypothesis follows, since, by construction, $C_{0}\left(S_{A}^{n}\right)=M_{0}\left(S_{A}^{n}\right)$.

Assume $C_{p-1}\left(S_{A}^{n}\right)=M_{p-1}\left(S_{A}^{n}\right)$. Consider a coefficient $c_{u, c}^{p}$ in $C_{p}\left(S_{A}^{n}\right)$. Let $F_{u, c}^{p}$ be the exclusive OR of all product terms whose coefficient in $R M_{p}\left(S_{A}^{n}\right)$ is $c_{u, c}^{p}$; i.e. $F_{u, c}^{p}$ is the exclusive OR of all product terms that have $u$ uncomplemented and $c$ complemented variables. We form an expression for $c_{u, c}^{p}$ as a function of coefficients in $C_{p-1}\left(S_{A}^{n}\right)$ by substituting $1 \oplus \bar{x}_{p}$ for $x_{p}$ in $R M_{p-1}\left(S_{A}^{n}\right)$. Such a substitution transforms $R M_{p-1}\left(S_{A}^{n}\right)$ into $R M_{p}\left(S_{A}^{n}\right)$.

From this last observation, we can write

$$
\begin{array}{r}
c_{u, c}^{p} F_{u, c}^{p}=\left.\left.c_{u, c}^{p-1} F_{u, c}^{p-1}\right|_{x_{p} \notin} \oplus c_{u+1, c}^{p-1} F_{u+1, c}^{p-1}\right|_{x_{p} \in} \\
\left.\oplus c_{u+1, c-1}^{p-1} F_{u+1, c-1}^{p-1}\right|_{x_{p} \in},
\end{array}
$$

where

$\left.c_{u, c}^{p-1} F_{u, c}^{p-1}\right|_{x_{p} \notin}$ represents all terms in $R M_{p-1}\left(S_{A}^{n}\right)$ that have $u$ uncomplemented and $c$ complemented variables and do not contain $x_{p}$ (i.e. substituting $1 \oplus \bar{x}_{p}$ for $x_{p}$ does not affect these terms),

$\left.c_{u+1, c}^{p-1} F_{u+1, c}^{p-1}\right|_{x_{p} \in}$ represents all terms in $R M_{p-1}\left(S_{A}^{n}\right)$ that have $u+1$ uncomplemented variables, including $x_{p}$, and $c$ complemented variables (i.e. these terms do not now have $x_{p}$ by virtue of substituting 1 of $1 \oplus \bar{x}_{p}$ ), and

$\left.c_{u+1, c-1}^{p-1} F_{u+1, c-1}^{p-1}\right|_{x_{p} \in}$ represents all terms in $R M_{p-1}\left(S_{A}^{n}\right)$ that have $u+1$ uncomplemented variables, including $x_{p}$, and $c-1$ complemented variables (i.e. these terms now have $\bar{x}_{p}$ by virtue of substituting $\bar{x}_{p}$ of $1 \oplus \bar{x}_{p}$ ).
We distinguish three cases.

1) $c=p$. For this value of $c$, (6) yields

$$
c_{u, p}^{p} F_{u, p}^{p}=\left.c_{u+1, p-1}^{p-1} F_{u+1, p-1}^{p-1}\right|_{x_{p} \in},
$$

since $c_{u, p}^{p-1}=c_{u+1, p}^{p-1}=0 . F_{u, p}^{p}=\left.F_{u+1, p-1}^{p-1}\right|_{x_{p} \in}$, and

$$
c_{u, p}^{p}=c_{u+1, p-1}^{p-1} .
$$

From (3) and (4),

$$
m_{u, p}^{p}=t_{0, n-p-u}=m_{u+1, p-1}^{p-1} .
$$

Since, by assumption, $m_{u+1, p-1}^{p-1}=c_{u+1, p-1}^{p-1}$, we can conclude that $m_{u, p}^{p}=c_{u, p}^{p}$.

2) $0<c<p$. We claim,

$$
c_{u, c}^{p}=c_{u+1, c-1}^{p-1} .
$$

On the contrary, if

$$
c_{u, c}^{p}=c_{u+1, c-1}^{p-1} \oplus 1,
$$

for each product term in $\left.F_{u+1, c-1}^{p-1}\right|_{x_{p} \in \text {, there is }}$ an identical one in exactly one of $\left.F_{u, c}^{p-1}\right|_{x_{p} \notin}$ and $\left.F_{u+1, c}^{p-1}\right|_{x_{p} \in}$. But, this is impossible; all product terms

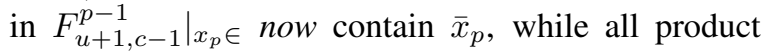
terms in $\left.F_{u, c}^{p-1}\right|_{x_{p} \notin}$ and $\left.F_{u+1, c}^{p-1}\right|_{x_{p} \in}$ do not now contain $x_{p}$. By an argument similar to that of Case 1), we can conclude that $m_{u, c}^{p}=c_{u, c}^{p}$, for $0<c<p$.

3) $c=0$. For this case, $\left.c_{u+1, c-1}^{p-1} F_{u+1, c-1}^{p-1}\right|_{x_{p} \in}=0$, and

$$
c_{u, 0}^{p} F_{u, 0}^{p}=\left.\left.c_{u, 0}^{p-1} F_{u, 0}^{p-1}\right|_{x_{p} \notin} \oplus c_{u+1,0}^{p-1} F_{u+1,0}^{p-1}\right|_{x_{p} \in} .
$$

No product term contains $x_{p}$; indeed, all product terms contain only uncomplemented variables. Since the function is symmetric, both $\left.F_{u, 0}^{p-1}\right|_{x_{p} \notin}$ and $\left.F_{u+1,0}^{p-1}\right|_{x_{p} \in}$ contain all combinations of all $u$ uncomplemented variables, as does $F_{u, 0}^{p}$. It follows that

$$
c_{u, 0}^{p}=c_{u, 0}^{p-1} \oplus c_{u+1,0}^{p-1} .
$$

By assumption, $m_{u, 0}^{p-1}=c_{u, 0}^{p-1}$ and $m_{u+1,0}^{p-1}=$ $c_{u+1,0}^{p-1}$. From (3) and (4), $m_{u, 0}^{p-1}=t_{p-1, n-p+1-u}$ and $m_{u+1,0}^{p-1}=t_{p-1, n-p-u}$. From (2),

$$
t_{p, n-p-u}=t_{p-1, n-p-u} \oplus t_{p-1, n-p+1-u} .
$$

Again, from (3) and (4),

$$
m_{u, 0}^{p}=t_{p, n-p-u}
$$

Therefore,

$$
m_{u, 0}^{p}=c_{u, 0}^{p}
$$




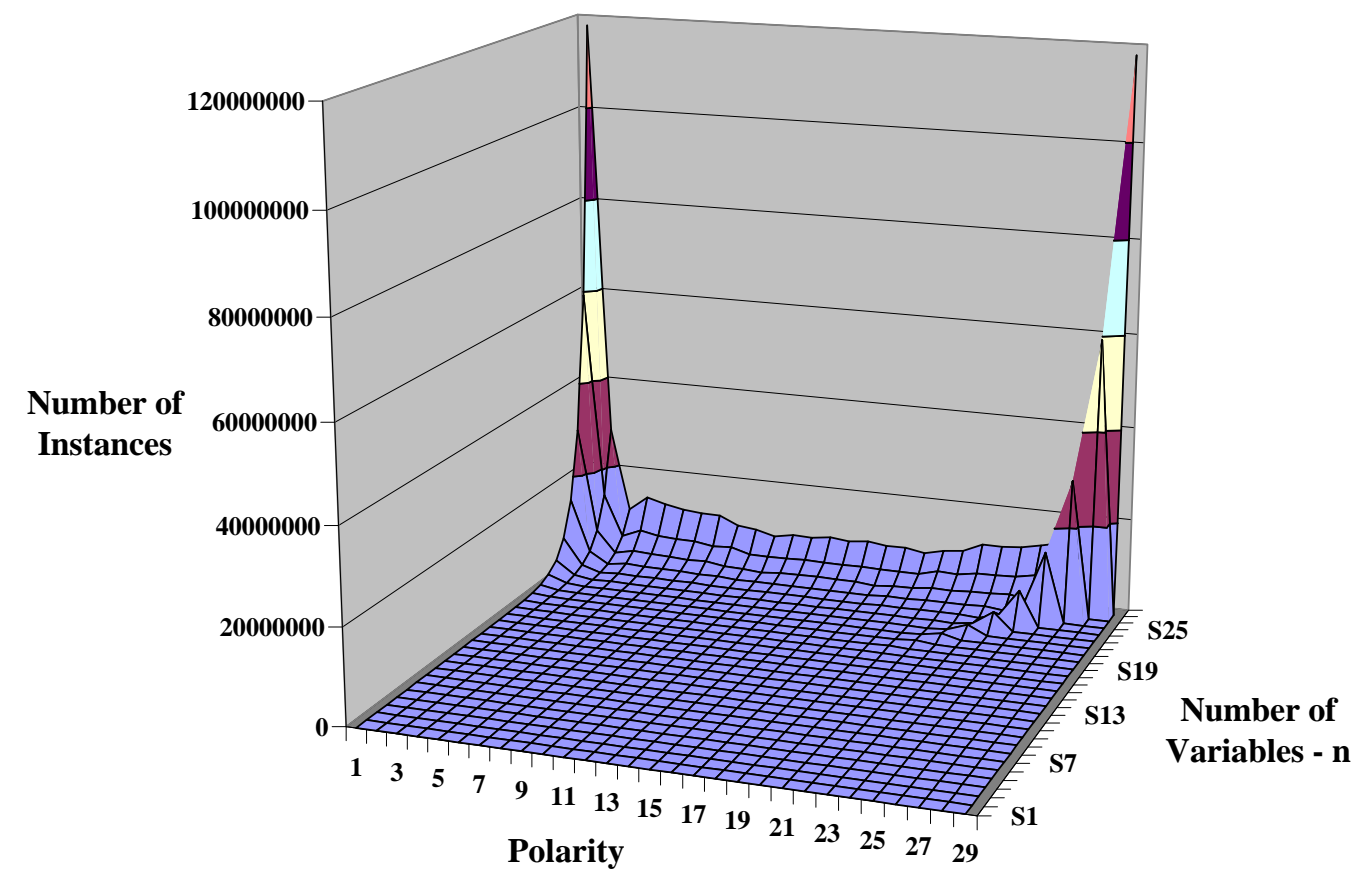

Figure 3. The distribution of optimal realizations of symmetric functions to polarity, for $2 \leq n \leq 28$.

Example 3.7 Consider the transeunt triangle $T\left(S_{\{0,1,2\}}^{3}\right)$, whose embedded submatrices are shown in Fig. 2. It can be seen that each $M_{p}\left(S_{\{0,1,2\}}^{3}\right)$ is identical to the corresponding coefficient matrix $C_{p}\left(S_{\{0,1,2\}}^{3}\right)$ shown in Example 2.4.

(End of Example)

Recall our remark earlier that the $R M_{p}\left(S_{A}^{n}\right)$ coefficients, in general, cannot be chosen arbitrarily. Since the coefficient matrix is embedded in the transeunt triangle, individual coefficients are related by the exclusive OR.

\section{Experimental Results}

\subsection{Number of Optimal Realizations ver- sus Polarity}

Because the transeunt triangle algorithm is so fast, we are able to make a remarkable observation about the distribution of symmetric functions to the polarities that optimally realize those functions. This is significant with respect to heuristics one might devise to minimize FPRM's. The relative merit of a heuristic for determining the best polarity to use in realizing a given symmetric function depends on the distribution of optimal realizations to polarity. A uniform distribution suggests that care is needed in making this choice. A nonuniform distribution, and particularly a polarity with a significantly higher propensity for optimal realizations suggests that less care is needed, or even that one should simply choose the favored polarity. Our next result is counterintuitive; it shows that the latter holds.

By exhaustively enumerating all transeunt triangles, we can determine how the number of optimal realizations are distributed to the various polarities. Table 1 shows this distribution for $n$ from 1 to 14 . That is, if a $n$-variable function is best realized by some polarity, it contributes 1 to the count shown in the row associated with $n$ and the column associated with that polarity. Certain functions contribute to more than one count. For example, in the first row, corresponding to $n=1$, three functions are shown as best realized by polarity 0 and three as best realized by polarity 1 , for a total of six. However, there are only four symmetric functions. Indeed, two functions, those with carry vectors [00] and [11], are optimally realized by both polarities. For large $n$, the data shows that only a small fraction of functions are best realized by more than one polarity.

Fig. 3 shows this data graphically, for $2 \leq n \leq 28$. It is interesting that polarities 0 and $n$ realize many more functions optimally than other polarities, as shown by the two ridges on each side. For example, for $n=28$, polarities 0 and 28 each realize $21.9 \%$ of the total number of functions. It should be noted that, for this value of $n$, only a small fraction of the functions have more than one optimal realization. That is, most of the functions have a unique optimal polarity.

It is also interesting that the table and plot are symmetric with respect to polarity. This is a consequence of the 

Table 1. Number of optimal realizations of symmetric functions in the Reed-Muller expansion versus
polarity and $n$, the number of variables.

\begin{tabular}{|c|c|c|c|c|c|c|c|c|c|c|c|c|c|c|c|}
\hline$n$ & 0 & 1 & 2 & 3 & 4 & 5 & 6 & 7 & 8 & 9 & 10 & 11 & 12 & 13 & 14 \\
\hline 1 & 3 & 3 & & & & & & & & & & & & & \\
\hline 2 & 5 & 3 & 5 & & & & & & & & & & & & \\
\hline 3 & 10 & 6 & 6 & 10 & & & & & & & & & & & \\
\hline 4 & 16 & 8 & 6 & 8 & 16 & & & & & & & & & & \\
\hline 5 & 26 & 11 & 12 & 12 & 11 & 26 & & & & & & & & & \\
\hline 6 & 51 & 14 & 13 & 15 & 13 & 14 & 51 & & & & & & & & \\
\hline 7 & 90 & 26 & 25 & 27 & 27 & 25 & 26 & 90 & & & & & & & \\
\hline 8 & 165 & 44 & 31 & 36 & 39 & 36 & 31 & 44 & 165 & & & & & & \\
\hline 9 & 312 & 89 & 44 & 64 & 67 & 67 & 64 & 44 & 89 & 312 & & & & & \\
\hline 10 & 604 & 146 & 76 & 82 & 113 & 119 & 113 & 82 & 76 & 146 & 604 & & & & \\
\hline 11 & 1,219 & 282 & 101 & 173 & 150 & 219 & 219 & 150 & 173 & 101 & 282 & 1,219 & & & \\
\hline 12 & 2,288 & 582 & 214 & 227 & 345 & 340 & 395 & 340 & 345 & 227 & 214 & 582 & 2,288 & & \\
\hline 13 & 4,411 & 1,148 & 364 & 659 & 467 & 703 & 596 & 596 & 703 & 467 & 659 & 364 & 1,148 & 4,411 & \\
\hline 14 & 8,578 & 2,296 & 687 & 1,087 & 1,226 & 891 & 1,165 & 1,251 & 1,165 & 891 & 1,226 & 1,087 & 687 & 2,296 & 8,578 \\
\hline
\end{tabular}

following.

Theorem 4.2 For each symmetric function $f(X)$ realized optimally in a Reed-Muller expansion using polarity $p$, for $0 \leq p \leq n$, there exists a symmetric function $f^{\prime}(X)$ that is realized optimally using polarity $n-p$.

\section{Proof}

Consider a p-polarity Reed-Muller expansion of $f(X)$ that is optimal. Complement all variables. The resulting expression is a Reed-Muller expansion of polarity $n-p$ of $f(\bar{X})$. Since the original expansion is optimal, so also is the modified one.

From Theorem 4.2, it follows that the distribution of the optimal polarities of symmetric functions is symmetric. That is, if a symmetric function $f(X)$ is optimally realized by polarity $p$, then the anti-dual function $f_{a}(X)$, obtained from $f(X)$ by complementing the all variables, is optimally realized by polarity $n-p$. It also implies that, if an optimal realization of some self-anti-dual function $f_{\text {sad }}(X)$ occurs with polarity $p$, then polarity $n-p$ is also an optimal polarity (a self-anti-dual function is invariant under complementation of all variables). If $p \neq n-p$, there are two distinct polarities optimally realizing the same symmetric self-antidual function; in Table 1, there is a contribution of 1 to the distribution from both polarities $p$ and $n-p$ from the same function.

\subsection{Percentage of Optimal Realizations versus $n$}

As observed in the previous two figures, many symmetric functions are optimally realized by polarities 0 and $n$. The exact percentage is shown in Table 2 . It can be seen

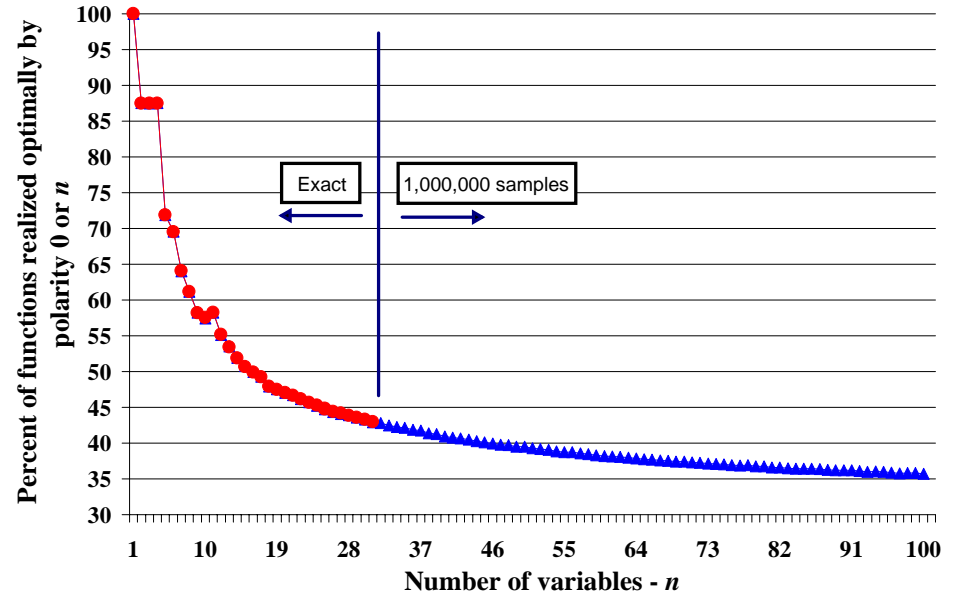

Figure 4. The percentage of functions realized optimally by polarity 0 or $n$ versus $n$.

that this percentage ranges from $100 \%$ for $n=1$ to below $40 \%$ for large $n$. The plot of this data is shown in Fig. 4. Along the horizontal axis is the number of variables $n$, where $1 \leq n \leq 100$, and along the vertical axis is the percentage of symmetric functions optimally realized either by polarity 0 or $n$. The figure shows two curves. The first, denoted by circles and labeled "Exact", is based on an exhaustive enumeration of all symmetric functions. The curve denoted by triangles and labeled "1,000,000 Samples" was produced by randomly generated symmetric functions. At $n=100,1,000,000$ samples represents only $3.95 \times 10^{-23} \%$ of the set of all symmetric functions. Even for this small percentage, the data is reasonably accurate, as shown by the consistency of adjacent data points. The fact that we were able to enumerate and analyze 1,000,000 samples of symmetric functions on 100 variables attests to the speed and 
Table 2. Percentage of $n$-variable symmetric functions optimally realized in a Reed-Muller expansion of polarity 0 or $n$. (Italicized values were computed using a sample set of $1,000,000$ example functions. Other values are exact.)

\begin{tabular}{||r|r||r|r||c|r||}
\hline \hline \multicolumn{5}{||c|}{$n=$ number of variables } \\
\multicolumn{2}{||c|}{$P_{0 / n}(n)=\%$ optimally realized by polarity 0 or $n$} \\
\hline \hline$n$ & $P_{0 / n}(n)$ & $n$ & $P_{0 / n}(n)$ & $n$ & $P_{0 / n}(n)$ \\
\hline \hline & & & & & \\
1 & $\mathbf{1 0 0 . 0 0}$ & 22 & $\mathbf{4 6 . 1 5}$ & 43 & 40.49 \\
2 & $\mathbf{8 7 . 5 0}$ & 23 & $\mathbf{4 5 . 6 7}$ & 44 & 40.25 \\
3 & $\mathbf{8 7 . 5 0}$ & 24 & $\mathbf{4 5 . 2 5}$ & 45 & 40.10 \\
4 & $\mathbf{8 7 . 5 0}$ & 25 & $\mathbf{4 4 . 8 0}$ & 46 & 39.94 \\
5 & $\mathbf{7 1 . 8 8}$ & 26 & $\mathbf{4 4 . 3 9}$ & 47 & 39.80 \\
6 & $\mathbf{6 9 . 5 3}$ & 27 & $\mathbf{4 4 . 1 5}$ & 48 & 39.72 \\
7 & $\mathbf{6 4 . 0 6}$ & 28 & $\mathbf{4 3 . 8 6}$ & 49 & 39.48 \\
8 & $\mathbf{6 1 . 1 3}$ & 29 & $\mathbf{4 3 . 5 5}$ & 50 & 39.48 \\
9 & $\mathbf{5 8 . 2 0}$ & 30 & $\mathbf{4 3 . 3 1}$ & 51 & 39.29 \\
10 & $\mathbf{5 7 . 5 2}$ & 31 & $\mathbf{4 2 . 9 9}$ & 52 & 39.17 \\
11 & $\mathbf{5 8 . 2 5}$ & 32 & 42.81 & 53 & 39.04 \\
12 & $\mathbf{5 5 . 1 4}$ & 33 & 42.50 & 54 & 38.86 \\
13 & $\mathbf{5 3 . 4 3}$ & 34 & 42.27 & 55 & 38.75 \\
14 & $\mathbf{5 1 . 8 9}$ & 35 & 42.13 & 56 & 38.71 \\
15 & $\mathbf{5 0 . 6 7}$ & 36 & 41.87 & 57 & 38.55 \\
16 & $\mathbf{4 9 . 9 1}$ & 37 & 41.74 & 58 & 38.46 \\
17 & $\mathbf{4 9 . 2 3}$ & 38 & 41.38 & 59 & 38.30 \\
18 & $\mathbf{4 7 . 8 9}$ & 39 & 41.27 & 60 & 38.20 \\
19 & $\mathbf{4 7 . 4 9}$ & 40 & 40.93 & 61 & 38.15 \\
20 & $\mathbf{4 7 . 0 3}$ & 41 & 40.79 & 62 & 38.08 \\
21 & $\mathbf{4 6 . 6 3}$ & 42 & 40.64 & 63 & 37.95 \\
\hline \hline
\end{tabular}

efficiency of the transeunt triangle.

\section{Concluding Remarks}

If the distribution of the number of optimally realized functions to polarity was uniform, we would observe a decrease in the percentage of all functions realized optimally by the extreme two polarities similar to that of Fig. 4. At $n=1$, the percentage would also be $100 \%$. However, it would steadily decrease to 0 , and at $n=100$, it would be about $2 \%$, not $35 \%$. This suggests that the extreme polarities, which exhibit an inherent symmetry (the term associated with each coefficient is symmetric), provide an efficiency in the realization of symmetric functions. It also suggests that a heuristic in which any symmetric function is realized by one of the two extremes will perform reasonably well.

\section{References}

[1] R. C. Born and A. K. Scidmore, "Transformation of switching functions to completely symmetric switching functions," IEEE Trans. on Comp., Vol. C-7, pp. 569$599,1968$.
[2] J. T. Butler, G. W. Dueck, S. Yanushkevich, and V. P. Shmerko, "Comments on 'Sympathy: Fast Exact Minimization of Fixed Polarity Reed-Muller Expansion for Symmetric Functions'," IEEE Trans. on ComputerAided Design, Vol. 19, No. 11, pp. 1386-1388, Nov. 2000.

[3] J. T. Butler, G. W. Dueck, S. N. Yanushkevich, and V. P. Shmerko, "On the number of generators for transeunt triangles," Discrete Applied Mathematics, pp. 309-316, 2001.

[4] M. Cohn, "Inconsistent canonical forms of switching functions," IRE Trans. Elect. Computers, pp. 284-285, 1962.

[5] M. J. Davio and P. Deschamps and A. Thayse, Discrete and Switching Functions, McGraw-Hill Int. Book Co., 1978.

[6] D. L. Dietmeyer, "Generating minimal covers of symmetric functions," IEEE Trans. on Computer-Aided Design Integrated Circuits and Systems, pp. 710-713, 1993.

[7] D. Green, Modern Logic Design, Addison-Wesley Publishing Company, 1986.

[8] D. H. Green, "Reed-Muller canonical forms with mixed polarity and their manipulations," IEE Proceedings, Pt. E, pp. 103-113, 1990.

[9] D. H. Green, "Reed-Muller expansions with fixed and mixed polarities over GF(4)," IEE Proceedings, Pt. E, pp. 380-388, 1990.

[10] P. K. Lui and J. C. Muzio, "Boolean matrix transforms for the minimization of modulo-2 canonical expansions," IEEE Trans. on Comp., pp. 342-347, 1992.

[11] A. Mukhopadhyay and G. Schmitz, "Minimization of exclusive-OR and logical-equivalence switching circuits," IEEE Trans. on Comp., pp. 132-140, 1970.

[12] D. L. Ostapko and S. J. Hong, "Generating test example for heuristic Boolean minimization," IBM J. Research and Development, pp. 459-464, 1974).

[13] S. M. Reddy, "Easily testable realizations for logic functions," IEEE Trans. on Comp., pp. 1183-1188, 1972.

[14] T. Sasao and Ph. W. Besslich, "On the complexity of Mod-2 sum PLA's," IEEE Trans. on Comp., Vol. C-29, No. 2, pp. 262-266, Feb. 1990.

[15] T.Sasao, Switching Theory for Logic Synthesis, Kluwer Academic Publishers, Norwell, MA, 1999. 
[16] J. Saul, "Logic synthesis for arithmetic circuits using the Reed-Muller representation," Proc. Euro. Conf. Design Automation, pp. 109-113, 1992.

[17] I. Schafer and M. A. Perkowski, "Multiple-valued input generalized Reed-Muller forms," Proc. of the Inter. Symp. on Multiple-Valued Logic, pp. 40-48, May 1991.

[18] C. E. Shannon, "A symbolic analysis of relay and switching circuits," AIEE Trans., pp. 713-723, 1938.

[19] V. P. Suprun, "Polynomial expression of symmetric Boolean functions," Izvestija AN USSR. Techn. Kibernetika (in Russian), No. 4, pp. 123-127, May 1985.

[20] V. P. Suprun, "Fixed polarity Reed-Muller expressions of symmetric Boolean functions," Proc. IFIP WG 10.5 Workshop on Application of the Reed-Muller Expansions in Circuit Design, pp. 246-249, 1995.

[21] S. N. Yanushkevich, D. M. Miller, V. P. Shmerko, and R. S. Stanković, Decision Diagram Techniques for Micro- and Nanoelectronic Design, Taylor \& Francis/CRC Press, Boca Raton, FL, 2006.

[22] S. N. Yanushkevich and V. P. Shmerko, Introduction to Logic Design, Taylor \& Francis/CRC Press, Boca Raton, FL, 2008. 